\title{
Madelung energy for random metallic alloys in the coherent potential approximation
}

\author{
Korzhavyi, P. A.; Ruban, Andrei; Abrikosov, I. A.; Skriver, Hans Lomholt
}

Published in:

Physical Review B

Link to article, DOI:

10.1103/PhysRevB.51.5773

Publication date:

1995

Document Version

Publisher's PDF, also known as Version of record

Link back to DTU Orbit

Citation (APA):

Korzhavyi, P. A., Ruban, A., Abrikosov, I. A., \& Skriver, H. L. (1995). Madelung energy for random metallic alloys in the coherent potential approximation. Physical Review B, 51(9), 5773-5780.

https://doi.org/10.1103/PhysRevB.51.5773

\section{General rights}

Copyright and moral rights for the publications made accessible in the public portal are retained by the authors and/or other copyright owners and it is a condition of accessing publications that users recognise and abide by the legal requirements associated with these rights.

- Users may download and print one copy of any publication from the public portal for the purpose of private study or research.

- You may not further distribute the material or use it for any profit-making activity or commercial gain

- You may freely distribute the URL identifying the publication in the public portal

If you believe that this document breaches copyright please contact us providing details, and we will remove access to the work immediately and investigate your claim 


\title{
Madelung energy for random metallic alloys in the coherent potential approximation
}

\author{
P. A. Korzhavyi \\ Department of Theoretical Physics, Moscow Institute of Steel and Alloys, Moscow, Russia
}

\author{
A. V. Ruban \\ Center for Atomic-scale Materials Physics and Physics Department, Technical University of Denmark, \\ DK-2800 Lyngby, Denmark \\ I. A. Abrikosov \\ Condensed Matter Theory Group, Physics Department, Uppsala University, S-75121 Uppsala, Sweden \\ H. L. Skriver \\ Center for Atomic-scale Materials Physics and Physics Department, Technical University of Denmark, \\ DK-2800 Lyngby, Denmark
}

(Received 31 May 1994; revised manuscript received 25 October 1994)

\begin{abstract}
Within the conventional single-site coherent potential approximation (CPA) used to calculate thermodynamic properties of random alloys, the effect of charge transfer is neglected. We discuss a number of recent models based on the same mathematical form but with a different prefactor $\beta$ which allow one to include charge-transfer effects in the framework of the CPA. We show how the models work in actual calculations for selected metallic alloy systems, $\mathrm{Al}-\mathrm{Li}, \mathrm{Li}-\mathrm{Mg}$, and $\mathrm{Ni}-\mathrm{Pt}$, which exhibit charge transfer. We find that the so-called screened impurity model $(\beta=1)$, which is derived completely within the mean-field single-site approximation, leads to the best agreement with experimental lattice parameter and mixing energy data for $\mathrm{Al}-\mathrm{Li}$ and $\mathrm{Li}-\mathrm{Mg}$ alloys. However, for the $\mathrm{Ni}-\mathrm{Pt}$ system exhibiting strong ordering tendency this model seems to overestimate the Madelung energy of the completely random alloy, and in this case the screened-CPA method $\left(\beta=\frac{1}{2}\right)$ gives more correct results. It is suggested that a comparison with the results obtained by the ConnollyWilliams method may be used to determine an optimal value for $\beta$ depending on the alloy under consideration.
\end{abstract}

\section{INTRODUCTION}

Modern $a b$ initio theory of the thermodynamic properties of random alloys is based on the single-site coherent potential approximation (SS-CPA) in conjunction with local density theory. ${ }^{1,2}$ One important problem with this approach is the general neglect of local environment effects. In particular, the conventional SS-CPA leads to complete neglect of the Madelung contribution to the one-electron potential of the alloy components and to the electrostatic energy, even in alloys with a considerable charge transfer. This is so because in the SS-CPA the effective medium surrounding each atom is charge neutral. The magnitude of a possible charge transfer in a solid depends on the way in which space is divided into geometrical cells. For instance, in the atomic sphere approximation (ASA) the charge transfer appears as a nonzero net charge $Q_{i}$ of component $i$ given by

$$
Q_{i}=Z_{i}-\int_{S_{\mathrm{ws}}} d^{3} r \rho_{i}
$$

where the integral is taken over the atomic sphere with radius $S_{\mathrm{WS}}, Z_{i}$ is the atomic number, and $\rho_{i}$ the electron density. In an exact treatment the total energy of a solid cannot depend on the particular partition of space and the application of an approximation such as the ASA must be justified by the accuracy with which it yields a total energy independent of cell sizes. Experience shows that for most ordered alloys the ASA has the required accuracy. However, the situation is completely different in the case of a disordered alloy because within the SSCPA one does not know the detailed charge distribution around a particular site. Hence, one must rely on approximations like the ASA and include the corresponding Madelung contribution to the potential and the total energy.

Recently, Magri et $a l .^{3}$ and Lu et $a l .^{4}$ examined the Madelung energy in random metallic alloys by means of their cluster expansion of electrostatic lattice energies. They found that the net charge on an atom in a metallic alloy depends linearly on the number of its nearest unlike neighbors. Since the atomic occupation of the lattice sites in any random alloy fluctuates, there should be a corresponding fluctuation in the net charges of the alloy components. In general, such fluctuations should lead to a nonzero Madelung energy in a random alloy and since the CPA as a mean-field theory does not take such fluctuations into account, Lu et al. ${ }^{4}$ came to the conclusion that charge-transfer effects could not be correctly described within the existing SS-CPA approaches.

A number of suggestions to correct this shortcoming of the SS-CPA has been put forward. One such attempt by 
Abrikosov et al. ${ }^{5}$ is based on the empirical observation that the net charge on an impurity atom in a metallic matrix is almost completely screened by the net charges of its nearest neighbors. ${ }^{6}$ The idea is to take these screening effects into account by means of screened Madelung contributions to the one-electron potentials and the total energy. Calculations based on this model for a number of systems ${ }^{5,7,8}$ showed that the simple correction dramatically improved the agreement between experimental alloy studies and, for instance, the calculated concentration dependence of ground state properties of random alloys.

Later, Johnson and Pinski ${ }^{9}$ suggested an approach which includes charge-correlation effects directly in the CPA. The mean-field version of this charge-correlated CPA, screened CPA (scr-CPA), exploited the empirical fact also found in Refs. 3, 4 that the net (or excess) charge on an atom in an alloy is a linear function of the number of its nearest unlike neighbors. Although the physical origin of the scr-CPA was different from that of the correction suggested by Abrikosov et al. ${ }^{5}$ it turned out that the two approaches were mathematically equivalent except for the fact that the Madelung energy given by Abrikosov et al. ${ }^{5}$ was twice the value obtained within the scr-CPA by Johnson and Pinski. ${ }^{9}$ It was therefore suggested $^{9}$ that the expression for the Madelung energy in Ref. 5 was in error owing to the neglect of doublecounting.

Before we discuss the different expressions for the Madelung energy in random alloys we would like to point out that in those CPA schemes which are based on the ASA one may circumvent the Madelung problem by choosing the atomic sphere radii of the alloy components so as to obtain charge-neutral spheres. ${ }^{10,11}$ In that case the Madelung contribution will vanish completely. However, if the sphere radii are too different, one may compromise the accuracy of the ASA and a careful consideration of the trade-off between the different sources of errors is required to make such a scheme work.

At present there exist four different suggestions for the Madelung energy of a completely random metallic alloy. If $Q_{A}$ and $Q_{B}$ are the net charges of the alloy components given in the ASA by Eq. (1), $c$ is the alloy concentration, $e$ the electronic charge, and $R_{1}$ the radius of the first coordination shell, the expressions may be written

$$
E_{M}=-\beta e^{2} c(1-c) \frac{\left(Q_{A}-Q_{B}\right)^{2}}{R_{1}}
$$

where the prefactor $\beta$ is defined by

$$
\beta= \begin{cases}0.0 & \text { in Refs. 1, 2 } \\ 0.65735 & \text { fcc in Ref. 12 } \\ 1.0 & \text { in Ref. 5 } \\ 0.5 & \text { in Ref. 9 }\end{cases}
$$

Which of these prefactors is correct and which should be used in SS-CPA calculations? The analysis of this question is the main goal of the present paper and we shall address it by discussing the physical arguments for each of the four models and subsequently compare the results of calculations on three representative metallic al- loy systems, i.e., Al-Li, $\mathrm{Li}-\mathrm{Mg}$, and $\mathrm{Ni}-\mathrm{Pt}$, which exhibit charge-transfer effects.

\section{MADELUNG ENERGY OF A COMPLETELY RANDOM ALLOY}

The Madelung energy per atom of a lattice of $N$ net point charges $Q_{i}$ may be expressed as

$$
E_{M}=\frac{e^{2}}{2 N} \sum_{i \neq j} \frac{Q_{i} Q_{j}}{R_{i j}}
$$

where $R_{i j}$ is the distance between site $i$ and $j$. In a random alloy $Q_{i} Q_{j}$ may be replaced by the configurational average

$$
\left\langle Q_{i} Q_{j}\right\rangle_{m}=\frac{1}{N Z_{m}} \sum_{i=1}^{N} \sum_{k=1}^{Z_{m}} Q_{i} Q_{k}
$$

where the second sum runs over sites in the $m$ th coordination shell containing $Z_{m}$ atoms. The Madelung energy in a random binary alloy $A_{c} B_{1-c}$ is then

$$
E_{M}=\frac{e^{2}}{2} \sum_{m=1}^{\infty} \frac{\left\langle Q_{i} Q_{j}\right\rangle_{m}}{R_{m}} Z_{m}
$$

where $R_{m}$ is the radius of the $m$ shell.

To describe configurational effects we introduce occupation numbers $\xi_{i}=1$ or 0 if site $i$ is occupied by atom $A$ or $B$, respectively. The average occupation number is the concentration of the $A$ element in the alloy, i.e., $\left\langle\xi_{i}\right\rangle=c$. In the case of a completely random alloy the occupation numbers for different sites are independent and $\left\langle\xi_{i} \xi_{j}\right\rangle=c^{2}+c(1-c) \delta_{i j}$.

\section{A. Conventional SS-CPA}

In the conventional SS-CPA it is assumed that all atoms of the same kind have the same net charge $Q_{A}$ or $Q_{B}$, which does not depend on the local environment. As a result,

$$
Q_{i}=\xi_{i} Q_{A}+\left(1-\xi_{i}\right) Q_{B},
$$

and it follows on account of the overall charge neutrality that

$$
\left\langle Q_{i} Q_{j}\right\rangle=\left\langle Q_{i}\right\rangle\left\langle Q_{j}\right\rangle=0
$$

and, hence, $E_{M}=0$. This approximation will work well in many cases, ${ }^{1,2}$ but if the charge transfer is appreciable, which is the case for the three systems considered here, it may even lead to qualitatively incorrect results. ${ }^{5,10,11}$

\section{B. Charge-correlated model}

In a real random alloy any atomic configuration is possible and atoms of the same type may have different local 
atomic environments. In contrast to the assumption of the conventional SS-CPA one would therefore expect the local electronic states and in particular the net atomic charges to fluctuate. Such fluctuations of the net charge should lead to additional contributions to the Madelung energy of the alloy.

To take such charge correlations into account, Lu et $a l .{ }^{4}$ evaluated the Madelung energy of a completely random metallic alloy by means of scheme which was based on the emprical evidence that the net charge of an alloy component in metallic alloys is proportional to the number of atoms of opposite type in the first coordination shell. Here, we rewrite their analytic result in a more convenient form in order to simplify the comparision with other models. In their scheme the net charge of site $i$ is determined by ${ }^{4}$

$$
Q_{i}=\xi_{i} \tilde{Q}_{A}+\left(1-\xi_{i}\right) \tilde{Q}_{B}-\frac{1}{Z_{1}} \sum_{k=1}^{Z_{1}}\left[\xi_{k} \tilde{Q}_{A}+\left(1-\xi_{k}\right) \tilde{Q}_{B}\right]
$$

where $\tilde{Q}_{A}$ and $\tilde{Q}_{B}$ are the average net charges of the $A$ and $B$ components in the alloy and the summation runs over the $Z_{1}$ atoms in the first coordination shell.

Substitution of (8) into (3) results in the following expression for the Madelung energy of a random alloy with charge correlation:

$$
E_{M}^{\mathrm{CC}}=-\beta e^{2} c(1-c) \frac{\left(\tilde{Q}_{A}-\tilde{Q}_{B}\right)^{2}}{R_{1}}
$$

where

$$
\beta=1-\sum_{m=1}^{4} \frac{\kappa_{m} \zeta_{m}}{2 \varrho_{m}}
$$

$\kappa_{m}=k_{m} / Z_{1}, k_{m}$ is the number of shared nearest neighbors between sites $i$ and $i+m, \zeta_{m}=Z_{m} / Z_{1}$, and $\varrho_{m}=R_{m} / R_{1}$. The first four values for $\kappa, \zeta$, and $\varrho$ which give a nonzero contribution to (10) are listed in Table I. The values for the $\beta$ factor in the fcc and bcc lattices are 0.65735 and 0.69155 , respectively.

TABLE I. Coordination parameters of the fcc and bcc lattices for the first four coordination shells $(m=1,4)$.

\begin{tabular}{cccccc}
\hline \hline Lattice & & \multicolumn{5}{c}{$m$} \\
\cline { 3 - 6 } & & 1 & 2 & 3 & 4 \\
\hline \multirow{4}{*}{ fcc } & $\kappa_{m}$ & $1 / 3$ & $1 / 3$ & $1 / 6$ & $1 / 12$ \\
& $\zeta_{m}$ & 1 & $1 / 2$ & 2 & 1 \\
& $n_{m}$ & $1 / 3$ & $1 / 6$ & $1 / 3$ & $1 / 12$ \\
& $\varrho_{m}$ & 1 & $\sqrt{2}$ & $\sqrt{3}$ & 2 \\
\hline \multirow{4}{*}{ bcc } & $\kappa_{m}$ & 0 & $1 / 2$ & $1 / 4$ & $1 / 8$ \\
& $\zeta_{m}$ & 1 & $3 / 4$ & $3 / 2$ & 1 \\
& $n_{m}$ & 0 & $3 / 8$ & $3 / 8$ & $1 / 8$ \\
& $\varrho_{m}$ & 1 & $2 / \sqrt{3}$ & $2 \sqrt{2} / \sqrt{3}$ & 2 \\
\hline \hline
\end{tabular}

\section{Screened impurity model}

It is intersting that the result of the charge-correlated model may be obtained without any statistical considerations, i.e., in the mean-field approximation. This allows us to describe charge-transfer effects completely within the framework of the SS-CPA as we shall explain in the following.

The basic idea behind the CPA is the substitution of the initial random alloy by an ordered lattice of effective scatterers. In the single-site approximation the properties of these effective atoms must be determined selfconsistently by the condition that the scattering of electrons off real atoms treated as impurities in the effective medium vanishes on the average. Hence, in the framework of the SS-CPA the attention is focused on a single atom embedded in the mean-field effective medium. If this atom has a nonzero net charge, one must decide what should be done with the compensating charge in order to make the whole system charge neutral. If, for instance, one assumes that the compensating charge is distributed equally over all sites in the effective medium, the Madelung potential for this atom and consequently also the corresponding Madelung energy is zero. Other models may, however, lead to nonzero Madelung potential and energy.

It appears difficult to devise a model for the compensating charge from a purely mathematical point of view. Instead, we shall base our considerations on the analysis of results obtained by more sophisticated methods which goes beyond the single-site approximation. In particular, we consider the results of self-consistent Green's function calculations of impurity atoms where the perturbation of the nearest neighbor atomic shells are taken into account. ${ }^{6}$ These calculations show that, at least for metallic systems, the perturbation in the charge density induced by the impurity atom is small beyond its nearest neighbor shell, and that practically all the compensating charge is located on the nearest neighboring atoms.

Based on this observation we suggest the following model which may be called the screened impurity model (SIM) for the Madelung contributions to the potential and the total energy of a random alloy. First, the net charge of an alloy component $Q_{A(B)}$ embedded in the effective CPA medium is completely screened by the first shell of its surrounding effective atoms. Second, the screening charge is uniformly distributed among all the $Z_{1}$ nearest neighbor atoms and the net charge on each nearest neighbor is

$$
Q_{1}=Q_{2}=\cdots=Q_{Z_{1}}=-\frac{Q_{A(B)}}{Z_{1}}
$$

The corresponding Madelung potential for the impurity atom is

$$
V_{M}^{A(B)}=-e^{2} \sum_{i=1}^{Z_{1}} \frac{Q_{i}}{R_{1}}=e^{2} \frac{Q_{A(B)}}{R_{1}}
$$

where the sum runs over the atoms in the first coordination shell, and the Madelung energy of the cluster con- 
sisting of the impurity atom plus the closest neighbors is

$$
E_{M}^{A(B)}=\frac{e^{2} Q_{A(B)}\left(\sum_{k=1}^{Z_{1}} Q_{k}\right)}{R_{1}}+\frac{e^{2}}{2} \sum_{i, j=1}^{Z_{1}} \frac{Q_{i} Q_{j}}{R_{i j}} .
$$

In (13) the first term is the energy of the electrostatic interaction between the impurity atom and its compensating charge and the second term is the electrostatic energy of the compensating charge.

If (11) is substituted into (13), we obtain

$$
E_{A(B)}=-\beta^{*} e^{2} \frac{Q_{A(B)}^{2}}{R_{1}}
$$

where

$$
\beta^{*}=1-\sum_{m=1}^{4} \frac{n_{m}}{2 \varrho_{m}}
$$

In (15) $n_{m}=N_{m} / Z_{1}$ and $N_{m}$ is the number of impurity nearest neighbors which are in the $m$ th coordination shell with respect to some impurity nearest neighbor.

Within the SIM the Madelung energy of a completely random alloy is then

$E_{M}^{\mathrm{SIM}}=c E_{M}^{A}+(1-c) E_{M}^{B}=-\beta^{*} e^{2} c(1-c) \frac{\left(Q_{A}-Q_{B}\right)^{2}}{R_{1}}$

and by means of the parameters listed in Table I and the fact that $n_{m}=\kappa_{m} \zeta_{m}$ one finds $\beta^{*}=\beta$. Hence, the charge-correlated model and the SIM, in the particular case where the compensating charge is uniformly distibuted over the nearest neighbor atoms, give the same Madelung energy. On the other hand, we do not consider the two models to be equivalent because the Madelung energy in the SIM does not depend on the type of atoms surrounding the impurity atom. Furthermore, in contrast to (9) the SIM expression (14) has been obtained without any consideration of correlation effects. In fact, (14) is derived completely within mean-field theory and may therefore be used in SS-CPA calculations.

It was suggested in Refs. 5, 8 that one may use the SIM expression with $\beta^{*}=1$ for the Madelung energy of a random metallic alloy, i.e.,

$$
E_{M}^{\mathrm{SS}-\mathrm{SIM}}=-e^{2} c(1-c) \frac{\left(Q_{A}-Q_{B}\right)^{2}}{R_{1}}
$$

in SS-CPA calculations, which means that one neglects the interactions between the screening charges themselves as represented by the last term in (15). Since a single-site contribution to the total energy should include only interactions between a given site and its neighbors, the neglect of the interneighbor interactions seems a natural consequence of the single-site approximation. As a matter of fact, it is impossible to obtain an exact expression for the $\beta^{*}$ factor in the single-site approximation, because the detailed inner structure of the screening charge is unknown. In this situation, the best choice of $\beta^{*}$ may be determined by a comparison of calculated properties with appropriate experimental data. It is our experience that (17) works well for different metallic alloy systems and as rule dramatically improves the agreement between calculated ground state properties and the experimental data.

One advantage of the SIM is the fact that one may generalize the correction to the Madelung potential and energy to the case of a multicomponent alloy. This is so because the Madelung potential in Eq. (12) only depends on the on-site net charge and therefore has the same form for any number of alloy components. Consequently, the Madelung energy in Eq. (16) may be obtained as

$$
E_{M}^{\mathrm{SIM}}=\sum_{i} c_{i} E_{M}^{i}
$$

where $c_{i}$ is a concentration of the $i$ th component, and $E_{M}^{i}$ is given by Eq. (14).

Within the so-called screened CPA Johnson and Pinski $^{9}$ have derived an expression for the Madelung energy of a random alloy which is equivalent to (14) but with $\beta^{*}=1 / 2$. In doing so, they apply an expression equivalent to (5) which implies the statistical equivalence of all the lattice sites. However, in their derivation the atom in a central site and its nearest neighbors are treated as statistically inequivalent because the excess charge on the central atom is assumed not to depend on the number of unlike nearest neighbors while at the same time such a dependence is included when the nearest neighbors are considered. It seems to us that a derivation of the Madelung energy within the scr-CPA should be based on the analytic charge-correlated model of Lu et al. ${ }^{4}$ described in Sec. II B. However, in that case we do not find an approximation which would lead to the result $\beta=1 / 2$.

In conclusion, we would point out that the SS-SIMCPA and the scr-CPA are based on different models for the Madelung energy of a random alloy and that they are obviously both approximate. One way to determine which is the better approximation is to compare the results of actual calulations with experimental observations. However, as always the most consistent comparison is with other first-principles calculations. In particular, one may compare with the mixing energy of a completely random alloy calculated by the Connolly-Williams method (CWM). ${ }^{13}$ The remainder of the present paper is devoted to such comparisons.

\section{DETAILS OF CALCULATION}

The present calculations have been performed by means of the tight-binding linear muffin-tin orbital (LMTO) method ${ }^{14-19}$ for the pure elements and by the LMTO-CPA method ${ }^{20,21}$ for the fcc Ni-Pt, fcc Al-Li, and bcc $\mathrm{Li}-\mathrm{Mg}$ alloys applying in all cases the second-order scalar-relativistic tight-binding LMTO Hamiltonian with $s, p$, and $d$ orbitals within the atomic sphere approximation. For exchange and correlation we used the Perdew- 
Zunger parametrization ${ }^{22}$ of the many-body calculations of Ceperley and Alder. ${ }^{23}$ The $k$-space integrals were calculated in the 1/48 part of the Brillouin zone over $240 k$ points for the fcc lattice and $280 k$-points for the bcc lattice. All the energy integrals of the LMTO-CPA method were evaluated by means of 16 points on a semicircular contour in the complex energy plane.

At each concentration we performed fully selfconsistent all-electron calculations for ten different values of the Wigner-Seitz radius around the equilibrium with a step equal to $0.05 \mathrm{a} . \mathrm{u}$. The convergence criteria for the total energy was $0.001 \mathrm{mRy}$. In order to determine the equilibrium lattice parameter and ground state energy we used the exponential function fit proposed by Moruzzi et al. ${ }^{24}$

Since the constituents in the $\mathrm{Al}-\mathrm{Li}$ and $\mathrm{Li}-\mathrm{Mg}$ alloy systems have different ground state crystal structures, we calculated the mixing energy rather than the mixing enthalpy. The former is defined by

$$
E_{\operatorname{mix}}^{\text {alloy }}(c)=E_{0}^{\text {alloy }}-c E_{0}^{A}-(1-c) E_{0}^{B}
$$

where $E_{0}^{j}$ with $j=$ alloy, $A$, or $B$ represents the ground state total energies of an alloy and its components determined at the equilibrium volume corresponding to the crystal structure of the alloy.

We have considered three different models: (i) the conventional SS-CPA without any contributions to the Madelung potential and energy, (ii) the SS-SIM-CPA with the Madelung potential given by (12) and the Madelung energy given by (17), and (iii) scr-CPA of Ref. 9 represented by (12) and (16) with $\beta=1 / 2$. The results of the calculations with neutral spheres as well as the results for equivolume spheres were taken from Refs. 10, 11. The latter corresponds to our conventional CPA calculations, and the small deviations from our results are probably due to the differences in computational details, e.g., the method used in the Brillouin zone integration.

\section{RESULTS}

\section{A. Al-Li and Li-Mg alloys}

The $\mathrm{Al}-\mathrm{Li}$ and $\mathrm{Li}-\mathrm{Mg}$ alloy systems have very unusual properties in the Al-rich and Li-rich regions, respectively. For instance, Al-Li exhibits a decrease in the lattice parameter [see Fig. 1(a)] and an increase in the Young's modulus with increasing $\mathrm{Li}$ concentration in spite of the fact that pure Li has a larger atomic volume and a smaller Young's modulus than pure $\mathrm{Al}^{8}$ The $\mathrm{Li}-\mathrm{Mg}$ system is interesting because of the peculiar concentration dependence of its thermodynamic and kinetic properties connected with an electronic topological transition. ${ }^{25,5}$ It has also been found experimentally that the lattice parameter of $\mathrm{Li}-\mathrm{Mg}$ alloys decreases with increasing $\mathrm{Mg}$ concentration in the Li-rich region [see Fig. 2(a)] although Vegard's law would predict an increase.

There are two reasons why these particular systems may serve in a test of different models for the Madelung

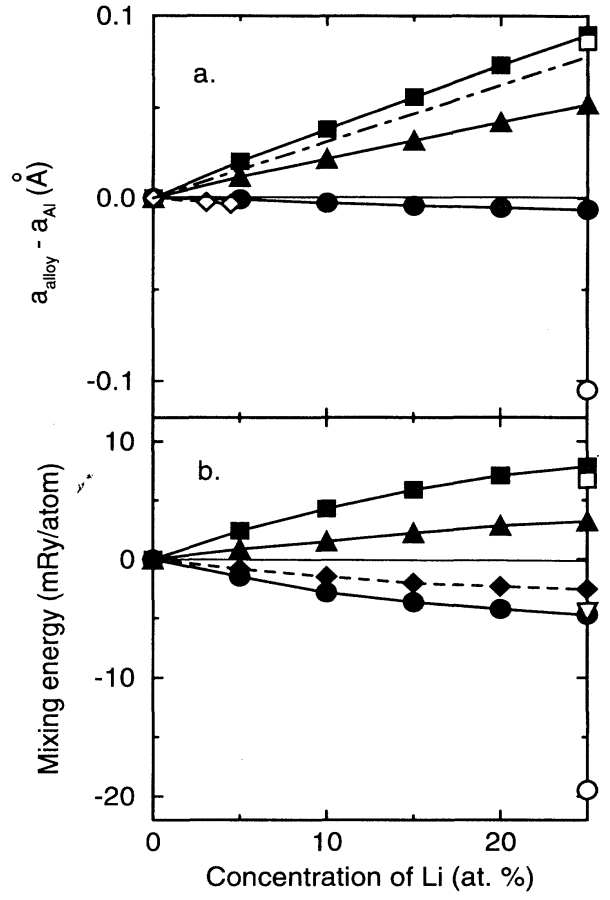

FIG. 1. The alloy lattice parameter relative to that of pure $\mathrm{Al}$ (a) and the mixing energy (b) in the Al-Li system plotted as functions of the $\mathrm{Li}$ concentration. The solid squares, circles, and triangles are the results of the conventional SS-CPA, the SS-SIM-CPA, and the scr-CPA calculations, respectively. The results obtained with equal (open square) and neutral (open circle) spheres at $25 \%$ are those of Ref. 11 . In (a) the dot-dashed line corresponds to Vegard's law and the experimental lattice parameters (open diamonds) are from Ref. 27. The open triangle in (b) is the mixing energy of the ordered $\mathrm{Al}_{3} \mathrm{Li}$ compound in the $\mathrm{L}_{2}$ structure calculated by the LMTO method. The solid diamonds are the Connolly-Williams results for the mixing energy of completely random Al-Li alloys.

energy in random alloys. First, as was noticed before ${ }^{5,8}$ their thermodynamic properties are very sensitive to charge-transfer effects which are considerable in these systems. Second, they do not exhibit any strong tendency towards ordering, and, moreover, there are practically no short-range-order (SRO) effects in the $\mathrm{Li}-\mathrm{Mg}$ alloys ${ }^{26}$ which makes possible a direct comparison between the properties calculated within the SS-CPA and the experimental values.

Here, we have considered fcc-based Al-Li alloys with up to $25 \%$ of $\mathrm{Li}$ and bcc-based $\mathrm{Li}-\mathrm{Mg}$ alloys with up to $70 \%$ of $\mathrm{Mg}$. The calculated lattice parameters are presented in Table II and it seen that the LMTO-ASA calculations underestimate the lattice constants for both pure $\mathrm{Al}$ and $\mathrm{Li}$, a trend one would also expect in the alloys. Therefore, when the conventional CPA calculations predict lattice parameters for random $\mathrm{Al}_{75} \mathrm{Li}_{25}$ and $\mathrm{Li}_{80} \mathrm{Mg}_{20}$ which appear to be in seemingly better agreement with the experiment values than the results of all other calculations, this is the result of a similar underestimate. 


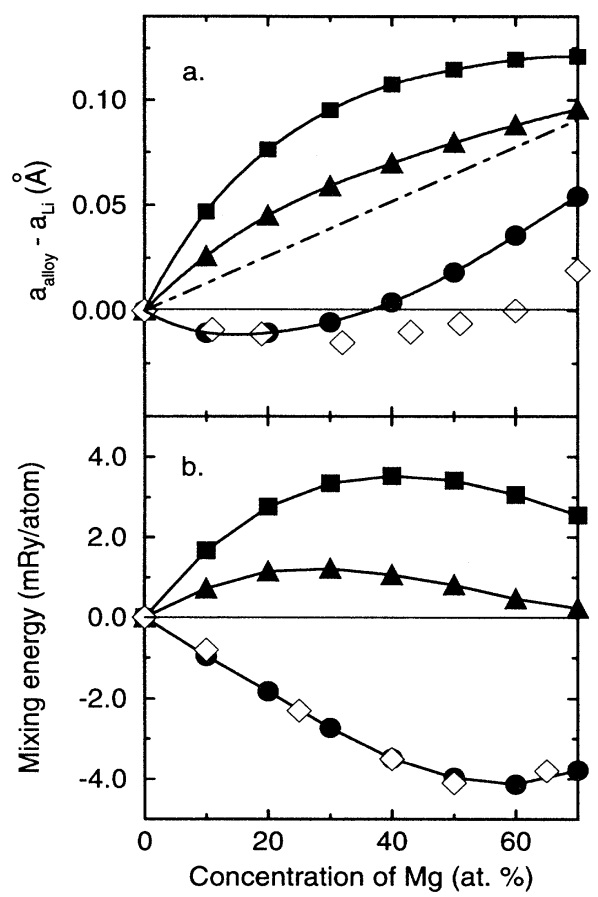

FIG. 2. The alloy lattice parameters relative to that of pure $\mathrm{Li}$ (a) and the mixing energy (b) in the Li-Mg system plotted as functions of the $\mathrm{Mg}$ concentration. Notation as in Fig. 1. The experimental results for the lattice parameter (Ref. 29) and the heat of formation of liquid $\mathrm{Li}-\mathrm{Mg}$ alloys (Ref. 31) are shown by open diamonds.

In fact, when we consider the concentration dependence of the lattice parameter calculated within the SSCPA the results turn out to be qualitatively incorrect; i.e., one finds in this case an increase of the lattice parameter in the $\mathrm{Al}-\mathrm{Li}$ as well as the $\mathrm{Li}-\mathrm{Mg}$ system as a function of the second component concentration [see Figs. 1(a) and $2(\mathrm{a})$ ] to the extent that both systems are expected to show a positive deviation from Vegard's law. On the other hand, if we include the SS-SIM correction to the
Madelung potential and energy, the calculated concentration dependences of the lattice parameters exhibit the experimetally observed trends.

We note that the neutral sphere calculation also qualitatively improves the agreement with experiment in the Al-Li alloys ${ }^{11}$ although the decrease of the lattice constant for the random $\mathrm{Al}_{75} \mathrm{Li}_{25}$ alloy relative to pure $\mathrm{Al}$ seems to be too large. In contrast, the scr-CPA leads to incorrect concentration dependences for $\mathrm{Al}-\mathrm{Li}$ as well as $\mathrm{Li}-\mathrm{Mg}$ alloys but corrects part of the failure of the conventional SS-CPA calculations. We take the close agreement between the SS-SIM-CPA calculations and the experimental lattice parameters shown in Figs. 1(a) and 2(a) to mean that the SIM provides an adequate description of charge-transfer effects, at least in the $\mathrm{Al}-\mathrm{Li}$ and $\mathrm{Li}-\mathrm{Mg}$ alloy systems.

We are not aware of any experimental values for mixing enthalpies of the solid state in the $\mathrm{Al}-\mathrm{Li}$ and $\mathrm{Li}-\mathrm{Mg}$ systems. However, as their alloys form stable solid solutions in a broad concentration range, one would expect negative values for the mixing energies. This expectation is partially supported by the heat-of-formation measurement of Mashkovetz and Puchkov for the liquid Li-Mg alloys $^{31}$ and for Al-Li alloys by a number of calculations for the ordered $\mathrm{Al}_{3} \mathrm{Li}$ compound ${ }^{11,32,33}$ predicting negative mixing energies.

The results presented in Figs. 1(b) and 2(b) show that the conventional CPA predicts positive mixing energies in both the $\mathrm{Al}-\mathrm{Li}$ and the $\mathrm{Li}-\mathrm{Mg}$ systems whereas the SIM-CPA leads to negative mixing energies as does in fact the single neutral sphere result ${ }^{11}$ for the $\mathrm{Al}_{75} \mathrm{Li}_{25}$ alloy. However, as in the case of the lattice parameter the neutral sphere approach seems to overestimate the effect of charge transfer. It is furthermore seen that the scr-CPA leads to concentration dependences which are intermediate between the results of the pure SS-CPA and those of the SIM-CPA and hence closer to the expected behavior. Since the Madelung term (17) in the SS-SIM is always negative, such a behavior is to be expected and it means that the conventional SS-CPA in the case of nonzero charge transfer will always overestimate mixing energies.

TABLE II. Lattice parameters (in $\AA$ ) for the $\mathrm{Ni}-\mathrm{Pt}$, Al-Li, and $\mathrm{Li}-\mathrm{Mg}$ systems.

\begin{tabular}{lccccccc}
\hline \hline System & LMTO & SS-CPA & SS-SIM-CPA & scr-CPA & $\begin{array}{c}\text { Equivolume } \\
\text { spheres }^{\mathrm{a}}\end{array}$ & $\begin{array}{c}\text { Neutral } \\
\text { spheres }^{\mathrm{a}}\end{array}$ & Experiment \\
\hline $\mathrm{Al}$ & 3.913 & & & & 3.919 & & $4.049^{\mathrm{b}}$ \\
$\mathrm{Al}_{95} \mathrm{Li}_{5}$ & & 3.933 & 3.912 & 3.925 & & & $4.046^{\mathrm{b}}$ \\
$\mathrm{Al}_{75} \mathrm{Li}_{25}$ & & 4.003 & 3.907 & 3.965 & 4.005 & 3.814 & $\begin{array}{c}4.010^{\mathrm{c}} \\
\mathrm{Li}\end{array}$ \\
$\mathrm{Li}$ & 3.356 & & & & & & $3.483^{\mathrm{d}}$ \\
$\mathrm{Ni}$ & 3.474 & 3.432 & 3.345 & 3.401 & & & $3.472^{\mathrm{d}}$ \\
$\mathrm{Pt}$ & 4.024 & & & & & & $3.517^{\mathrm{e}}$ \\
$\mathrm{Ni}_{50} \mathrm{Pt}_{50}$ & & 3.830 & 3.775 & 3.809 & 3.860 & 3.768 & $3.749^{\mathrm{e}}$ \\
\hline \hline
\end{tabular}

${ }^{a}$ Reference 11.

${ }^{b}$ Reference 27 , data for a random alloy at 4.47 at. \% Li.

${ }^{c}$ Reference 28, data for an ordered $\mathrm{Al}_{3} \mathrm{Li}$ compound.

${ }^{\mathrm{d}}$ Reference 29 , data for a random alloy at 19 at. $\% \mathrm{Mg}$.

e Reference 30. 
One must be careful when comparing calculated mixing energies valid for completely random alloys with experimental values which could exhibit short-range-order effects. While the Li-Mg alloys may safely be considered as completely random ${ }^{26}$ there appears to be a certain amount of SRO effect in the Al-Li system to the extent that a metastable ordered $\mathrm{Al}_{3} \mathrm{Li}$ phase $\left(L 1_{2}\right.$ structure) is formed. In the latter case a comparison with experiment may therefore be misleading.

As an alternative to the CPA calculations and to avoid the influence of SRO we have estimated the heat of formation of completely random Al-Li alloys on the basis of the CWM. ${ }^{13}$ Hence, we have performed LMTO calculations of the total energies of ordered $\mathrm{Al}-\mathrm{Li}$ alloys $\left(\mathrm{Al}, \mathrm{Al}_{3} \mathrm{Li}\right.$, $\mathrm{AlLi}, \mathrm{Li}_{3} \mathrm{Al}, \mathrm{Li}$ ) and extracted the Connolly-Williams interactions. These were subsequently used in the calculations of the mixing energies of completely random alloys whereby the Madelung contribution per definition was treated exactly. We conclude on the basis of the close agreement between the SS-SIM-CPA and the ConnollyWilliams calculations of the mixing energies shown in Fig. 1(b) that the SS-SIM-CPA provides an adequate description of charge-transfer effects in completely random $\mathrm{Al}-\mathrm{Li}$ and $\mathrm{Li}-\mathrm{Mg}$ alloys.

\section{B. Ni-Pt alloys}

The Ni-Pt alloys form fcc-based solid solutions in the complete concentration range at high temperatures, and exhibit a strong tendency towards ordering. In this system the conventional CPA calculations predict too large positive mixing enthalpies ${ }^{10,11}$ and the main reason for this failure, as suggested in Ref. 10, appears to be the neglect of charge-transfer effects in the SS-CPA. The Ni-Pt alloy system may therefore serve as a test of the applicibility of models for the Madelung potential and energy in the SS-CPA. However, one should keep in mind that the actual experimental data correspond to alloys with a certain amount of SRO effects as well as local atomic distortions while in the SS-CPA calculations one considers completely random alloys on a rigid perfect lattice.

The lattice parameters of pure $\mathrm{Ni}$, pure $\mathrm{Pt}$, and a $\mathrm{Ni}_{50} \mathrm{Pt}_{50}$ alloy obtained by various methods are shown in Table II. Here, one observes that all the calculated values agree with the experimental results to within a few percent (a maximum deviation of $3 \%$ is found for pure $\mathrm{Pt}$ ) and one cannot judge which of the computational techniques leads to the best overall agreement with the experiment results. The situation changes when we consider the concentration dependence of the lattice parameter in NiPt alloys shown in Fig. 3(a). Here, one sees that the conventional SS-CPA calculations, which neglect charge-transfer effects completely, strongly overestimate the deviation from Vegard's law. In the scr-CPA calculations the deviation from Vegard's law is somewhat reduced but still significant. In contrast, the SS-SIM-CPA results are in excellent agreement with the experimental observations and so is the single result of the neutral sphere approach. ${ }^{10,11}$

If we consider the mixing energies shown in Fig. 3(b)

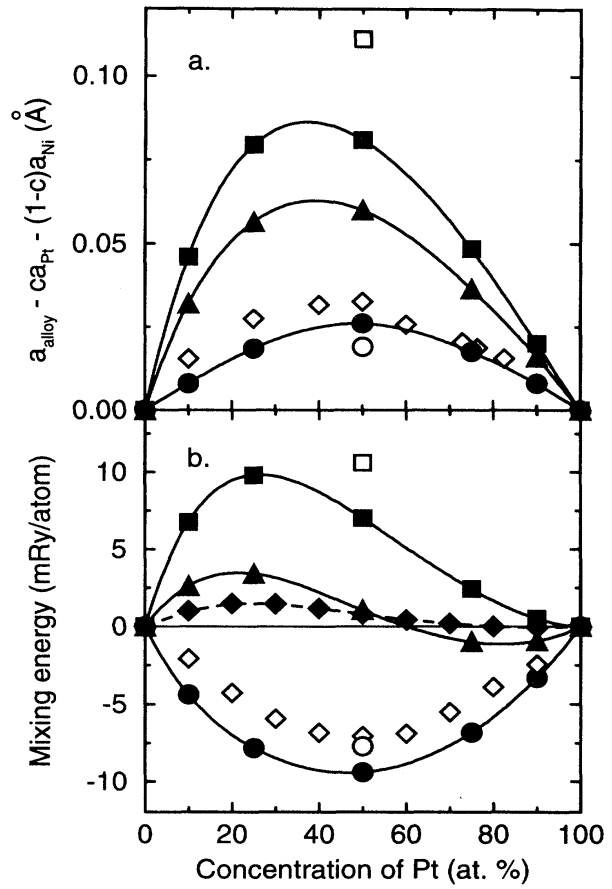

FIG. 3. The lattice parameter relative to Vegard's law (a) and the mixing energy (b) for the Ni-Pt system plotted as functions of the Pt concentration. Notation as in Fig. 1. The solid diamonds denote the CWM results from Ref. 35 . The experimental results for the lattice parameter (Ref. 30) and the mixing energy (Ref. 34) are shown by open diamonds.

we observe a similar pattern. In fact, the conventional CPA predicts positive mixing energies over the whole concentration range, while the SS-SIM-CPA model leads to negative mixing energies in complete agreement with the experimental data and the neutral sphere calculation. Again the scr-CPA is intermediate between the two. However, the experimental mixing energy for the $\mathrm{Ni}$ $\mathrm{Pt}$ system includes a short-range-order contribution and when such a contribution is included in the CWM calculations of Amador and Bozzolo ${ }^{35}$ also shown in Fig. 3(b) one in fact obtains a fit to the experimental data similar to that of the SIM calculation.

It appears that the success of the SIM may be fortuitous in the $\mathrm{Ni}-\mathrm{Pt}$ system since the model is expected to describe completely random alloys and not include any short-range-order effects. It should therefore not yield mixing energies in agreement with the experimental data. If we allow the prefactor $\beta$ of the SIM to vary and in particular to take on the value which gives the best fit to CWM calculations, we will ascertain that the subsequent SS-CPA calculations do not include modeldependent contributions which act as unwanted shortrange-order terms. Judged from the results in Fig. 1(b) and Fig. 3(b), $\beta$ values close to 1 and $1 / 2$ would lead to perfect agreement with the CWM calculations in the Al$\mathrm{Li}$ and the $\mathrm{Ni}-\mathrm{Pt}$ systems, respectively. We are at present investigating the feasibility of such a procedure. 


\section{SUMMARY}

We have compared a number of models for the Madelung energy of completely random metallic alloys within the atomic sphere approximation and the singlesite CPA, and found that all the existing models may be viewed in terms of a Madelung-type expression (2) where a particular model may be distinguished by its value of the prefactor $\beta$. We discuss two approximations which are derived entirely within the mean-field approximation but differ by a factor of 2 in the prefactor. The first model is based on the observation that the screening of an impurity in a metal occurs essential within the first coordination shell and this leads to a prefactor $\beta=1$ (SS-SIM-CPA). The second model explicitly includes the correlations caused by the distribution of nearest neighbor net charges and leads to $\beta=0.5$ (scr-CPA). ${ }^{9}$

We find in actual calculations that the difference in the prefactor $\beta$ strongly influences the calulated thermodynamic properties and suggest that $\beta$ for a particular alloy system may be determined in a fit to the results from calculations by the Connolly-Williams method. In the alloy systems treated here we would choose $\beta$ slightly less than 1 in $\mathrm{Al}-\mathrm{Li}$ and $\mathrm{Li}-\mathrm{Mg}$ and slightly larger than 0.5 in Ni-Pt. This means that charge-transfer effects cannot be neglected when the random alloy is considered and that the effect may be treated approximately within the single-site CPA.

The fact that $\beta$ may depend on the actual system under consideration should perhaps not come as a surprise. Both the SS-SIM-CPA and the scr-CPA models of charge-transfer effects as well as the charge-correlated model ${ }^{4}$ are approximations where one neglects the inner structure of the screening charge and imply that all the screening charge is located in the nearest neighbor shell. On the basis of the present calculations a value between 0.5 and 1 is expected.

\section{ACKNOWLEDGMENTS}

The Center for Atomic-scale Materials Physics is sponsored by the Danish National Research Foundation. The work of I.A.A. and A.V.R. was in part supported by The Swedish Material Consortium number 9 and grant number MQQ000 from the International Science Foundation, respectively.
${ }^{1}$ D. D. Johnson, D. M. Nicholson, F. J. Pinski, B. L. Gyorffy, and G. M. Stocks, Phys. Rev. Lett. 56, 2088 (1986).

2 D. D. Johnson, D. M. Nicholson, F. J. Pinski, B. L. Gyorffy, and G. M. Stocks, Phys. Rev. B 41, 9701 (1990).

${ }^{3}$ R. Magri, S.-H. Wei, and A. Zunger, Phys. Rev. B 42, 11388 (1990).

${ }^{4}$ Z. W. Lu, S.-H. Wei, A. Zunger, S. Frota-Pessoa, and L. G. Ferreira, Phys. Rev. B 44, 512 (1991).

${ }^{5}$ I. A. Abrikosov, Yu. H. Vekilov, P. A. Korzhavyi, A. V. Ruban, and L. E. Shilkrot, Solid State Commun. 83, 867 (1992).

${ }^{6}$ R. Zeller, J. Phys. F 17, 2123 (1987).

${ }^{7}$ S. I. Simak, A. V. Ruban, and Yu. H. Vekilov, Solid State Commun. 87, 393 (1993).

${ }^{8}$ P. A. Korzhavyi, A. V. Ruban, S. I. Simak, and Yu. Kh. Vekilov, Phys. Rev. B 49, 14229 (1994).

${ }^{9}$ D. D. Johnson and F. J. Pinski, Phys. Rev. B 48, 11553 (1993).

${ }^{10}$ P. P. Singh, A. Gonis, and P. E. A. Turchi, Phys. Rev. Lett. 71, 1605 (1993).

${ }^{11}$ P. P. Singh and A. Gonis, Phys. Rev. B 49, 1642 (1994).

12 The constant in Eq. (4.22) of Ref. 4 is given by $2 \beta Z_{1}^{2} / 16^{2}=$ $1.125 \beta$ where $Z_{1}$ is the coordination number in the first coordination shell of the fcc structure.

13 J. W. D. Connolly and A. R. Williams, Phys. Rev. B 27, 5169 (1983).

14 O. K. Andersen, Phys. Rev. B 12, 3060 (1975).

15 O. Gunnarsson, O. Jepsen, and O. K. Andersen, Phys. Rev. B 27, 7144 (1983).

${ }^{16}$ H. L. Skriver, The LMTO Method (Springer, Berlin, 1984).

${ }^{17}$ O. K. Andersen and O. Jepsen, Phys. Rev. Lett. 53, 2571 (1984).

18 O. K. Andersen, O. Jepsen, and D. Glötzel, in Highlights of Condensed-Matter Theory, edited by F. Bassani, F. Fumi, and M. P. Tosi (North-Holland, New York, 1985).
19 O. K. Andersen, Z. Pawlowska, and O. Jepsen, Phys. Rev. B 34, 5253 (1986).

${ }^{20}$ I. A. Abrikosov, Yu. H. Vekilov, and A. V. Ruban, Phys. Lett. A 154, 407 (1991).

${ }^{21}$ A. I. Abrikosov and H. L. Skriver, Phys. Rev. B 47, 16532 (1993).

22 J. Perdew and A. Zunger, Phys. Rev. B 23, 5048 (1981).

${ }^{23}$ D. M. Ceperley and B. J. Alder, Phys. Rev. Lett. 45, 566 (1980).

${ }^{24}$ V. L. Moruzzi, J. F. Janak, and K. Schwarz, Phys. Rev. B 37, 790 (1988).

${ }^{25}$ A. A. Varlamov, V. S. Egorov, and A. V. Pantsulaya, Adv. Phys. 38, 469 (1990).

${ }^{26}$ F. H. Herbstein and B. L. Averbach, Acta Metall. 4, 414 (1956).

${ }^{27}$ S. H. Kellington, D. Loveridge, and J. M. Titman, Br. J. Appl. Phys. (J. Phys. D) ser. 2, 2, 1169 (1969).

${ }^{28} \mathrm{~S}$. Kang and N. J. Grant, in Aluminum-Lithium II, edited by E. A. Starke, Jr. and T. H. Sanders (The Matallurgical Society of AIME, New York, 1983), p. 469.

${ }^{29}$ F. H. Herbstein and B. L. Averbach, Acta Metall. 4, 407 (1956).

${ }^{30}$ W. B. Pearson, A Handbook of Lattice Spacing and Structure of Metals and Alloys (Pergamon Press, London, 1967).

${ }^{31}$ V. P. Mashkovetz and L. V. Puchkov, Zh. Prikl. Khim. (Moscow) 38, 1875 (1965).

${ }^{32}$ X.-Q. Guo, R. Podloucky, J.-H. Xu, and A. J. Freeman, Phys. Rev. B 41, 12432 (1990).

${ }^{33}$ K. I. Masuda-Jindo and K. Terakura, Phys. Rev. B 39, 7509 (1989).

${ }^{34}$ R. Hultgren, P. D. Desai, D. T. Hawkins, M. Gleiser, and K. K. Kelley, Selected Values of Thermodynamic Properties of Binary Alloys (American Society for Metals, Metals Park, OH, 1973).

${ }^{35}$ C. Amador and G. Bozzolo, Phys. Rev. B 49, 956 (1994). 